\title{
Congenital Diaphragmatic Hernia presenting as recurrent respiratory tract infections
}

\author{
${ }^{1}$ Manori Prasadani, ${ }^{1}$ Sathiadas MG \\ ${ }^{\text {I}}$ Professorial Paediatric Unit, Teaching Hospital Jaffna.
}

\begin{abstract}
Congenital diaphragmatic hernia is a defect in the fetal diaphragm, allowing the contents of the abdominal cavity to protrude into the thorax. It is characterized by varying degree of pulmonary hypoplasia associated with decrease in cross sectional area of pulmonary vasculature. The case report presents an incidental finding of a congenital diaphragmatic hernia at an age of two years and four months, emphasizing the increased importance of thorough clinical examination that may lead you to an important incidental diagnosis.
\end{abstract}

\section{Introduction}

Congenital diaphragmatic hernia is a very challenging condition, commonly associated with high mortality due to pulmonary hypoplasia, pulmonary hypertension and co existing other anomalies (1). There fore the outcome of surgery depends on both diaphragmatic hernia and severity of coexisting anomalies. The diaphragm starts developing during the $6^{\text {th }}$ week of gestation as the septum transversum, and then the muscle fibers migrate into the membrane to form the diaphragm (2). Failure of this process produces congenital hernia. The commonest is the posterolateral defect or Bordalek Hernia. Less common are the anterolateral or Morgagni hernia. Most defects are sporadic. If the defect is severe and associated with other congenital abnormalities the lesion will be detected in Antenatal Anomaly Scan (e.g. Trisomy 13 h8/21, Donna - Barrow, Fryns syndrome) (3). If possible fetal interventions or newborn resuscitation followed by corrective surgery will be the ideal management. There will be a fraction of patients who go undetected, either because the defect is small and compensated or due to lack of high degree of suspicion medically and thorough clinical examination (4).

\section{Case Report}

Two years and four months old child with a normal birth history but having failure to thrive with recurrent respiratory tract infections, severe gastro oesophageal reflux presented to casualty ward with Lower respiratory tract infection. In the past he was treated once in two months with oral antibiotics for respiratory symptoms. The child weighed $10 \mathrm{~kg}(<-3 \mathrm{SD})$, Height $36 \mathrm{~cm}(<-3 \mathrm{SD})$ and BMI $19(<-3$ SD)

Respiratory system examination in the ward reveled; tracheal deviation to the right, apex beat not clearly felt, chest expansion reduced in the left side, vocal resonance reduced on the left side, percussion dull on left side and reduced air entry on left side. A chest $x$ ray was ordered and showed left sided diaphragmatic hernia with collapsed left lung, shifting the mediastinum to the right.

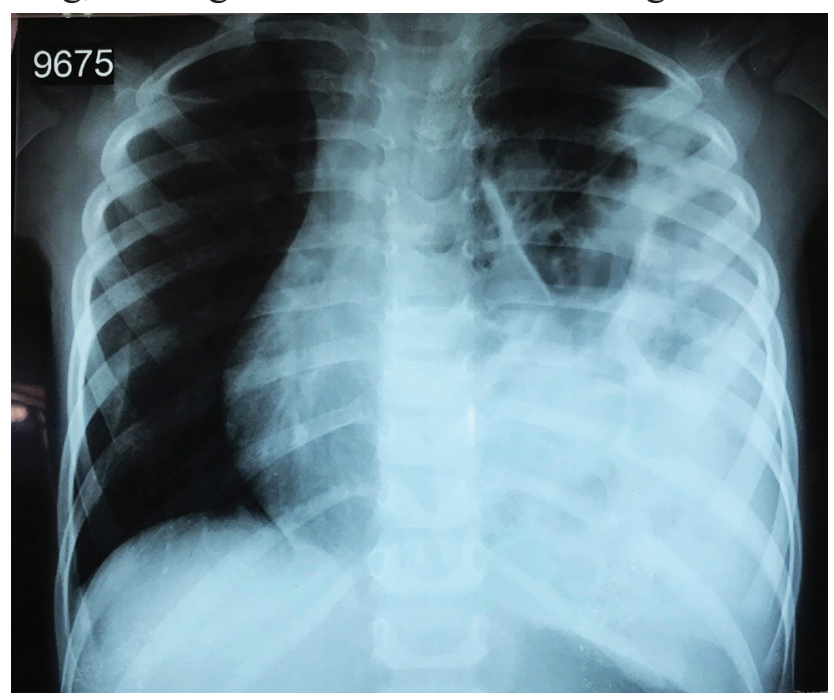


Ultra sound scan chest confirmed presence of bowel loops and the spleen in the left thorasic cavity. Chest infection was treated and child referred to peadiatric surgery team. He underwent corrective surgery at the specialized surgical unit for children.

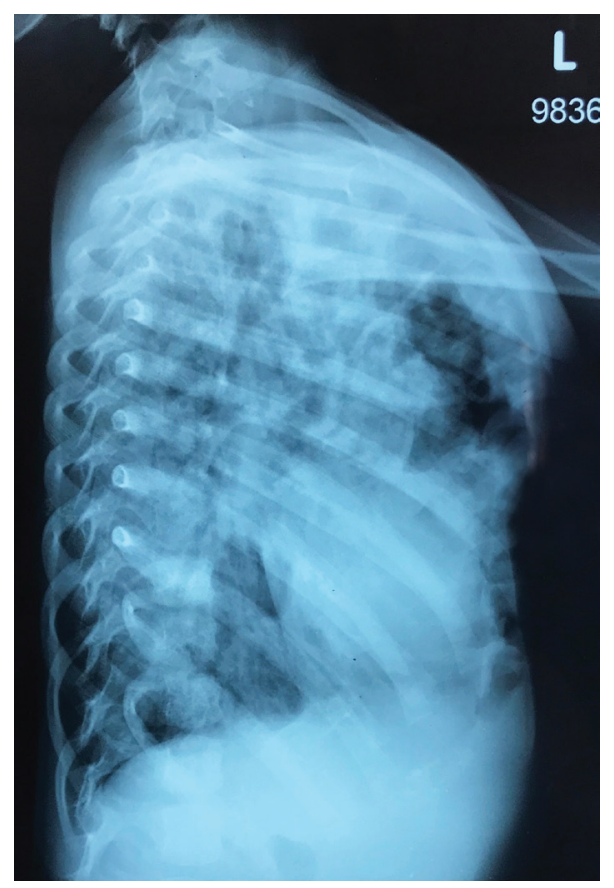

\section{Discussion}

The child having failure to thrive with gastrooesophageal reflux and recurrent chest infections can all be explained by the presence of this congenital diaphragmatic hernia. This is a good example of a congenital diaphragmatic hernia presenting in grown up child, where we expect to see it antenatally or in a newborn examination. A grown up child will present with multiple associated symptoms. Every child needs to be examined thoroughly during a visit to the hospital and investigated accordingly to look for missed or new underlying pathologies that may be the cause of this presenting complaint.

\section{Ethical consideration}

Consent was taken from the parents to publish the photograph of the child.

\section{Reference:}

1. Gujar, A., Rodrigues, D. D., Patil, K., Tambe, U., Sinha, S., \& Bhushan, A. (2013). Rare case report - congenital diaphragmatic hernia presentation in adult. The Indian journal of surgery, 75(Suppl 1), 44-46. https://doi. org/10.1007/s12262-011-0321-7

2. Nelson Text Book of Peadiatrics. Kliegmn R, Stanton B, St. Gene J, Sehor N.

3. Neil Melntosh, Peter J Helms, Rosalind L Smith.Congenital Diaphragmatic Hernia European Respiratory Journal 2012.

4. Henry Os Gabra, David J Wilson, Paul D Lasty. Management of Congenital Diaphragmatic Hernia. Peadiatric Child Health Journal 2018. 